
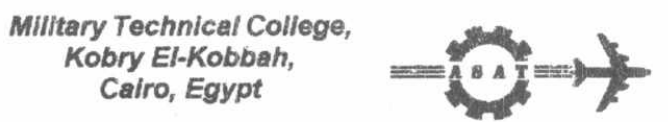

$9^{\text {th }}$ International Conference

On Aerospace Sciences \& Aviation Technology

\title{
DESIGN OF MAGNETIC / ELECTROMAGNETIC CONTROLLER USED FOR VIBRATION CONTROL. OF PLATES
}

\author{
Omer" A. A., Sherif" H. A. and Abdalla* A. E.
}

\begin{abstract}
In the last two decades, different actuators like piezoelectric, piezoceramic...etc are used for vibration control of beams, plates and shells. These actuators are suitable for control of vibration with small deflections, which require small amount of control force. Recently, the electromagnetic actuators are used for vibration control of bending moment and shear strain of these structures. In cases, which require a large deflection and control force, the electromagnetic actuators are more suitable. in the present study a magnetic-electromagnetic type actuator is used for control of lateral vibration of plates. The operating principle of the actuator depends on the attraction or repulsion forces that exist between the two magnets in lateral direction. The theory of electro-magnetic actuator is derived using the classical electromagnetic theory. A finite element model was developed and augmented into the compression finite element model [1]. The theoretical predictions of the performance of the controller are validated experimentally. Good agreement is obtained between the theoretical and experimental results.
\end{abstract}

\section{Introduction}

Active Constrained Layer Damping treatment (ACLD) is widely used recently for vibration damping of beams, plates and shell using piezo-electric sensor/actuator[2]. However, this method is suitable for control of vibration with small deflections which require small amount of control force, it is very difficult to be applied in cases, which require a large deflection and control force. This means other method is required for vibration damping of structures with large deflection. Zheng, et al. [3] have studied the vibration control of plate using moment type electromagnetic actuators which consists of rectangular permanent magnetic chips and a cylindrical coil actuator for control the bending moment of plates. The analysis performed was based on electromagnetic theory, and the relation between the control force and the control current has been obtained. They also found that if three actuators were properly oriented, it would be possible to perform deflection control, whether as cylindrical bending or twisting. Frangos, et al. [4] study the current controller design for an electromagnetic actuator using an on-line parameter optimization approach and they

\footnotetext{
- Egyptian Armed Forces
} 
that the controlled system has sever nonlinearites. This nonlinearites are due to nonlinear electromagnetic force, a nonlinear variation of inductance with position and force and a limited control voltage. The main advantage of their on-line optimization is the system nonlinearites are directly incorporated in the current controller design and the effectiveness of on-line parameter optimization depends on the selection of an appropriate controller structure and optimization function for the specific application at hand. However, this design utilize only electromagnetic actuators and give good results, the vibration control problem becomes very difficult if the power shut off, this is because the design can not work as passive controller. Baz [5] found a new surface treatment called Magnetic Constrained Layer Damping (MCLD) that provides effective means for attenuating undesirable structural vibrations. That treatment acts as smart damping material which consists of integrated arrays of constrained viscoelastic layer controlled passively by a specially arranged network of permanent magnets. The interaction between the magnets and viscoelastic layers enhance the energy dissipation of damping treatment by increasing the shear strain. Although that arrangement does not need electronics or circuits and have good results with vibration damping, there still need to another design for controlling plate with high vibration amplitude. In the present work, a new approach is introduced in which the electro-magnetic actuators are used as active means for controlling the lateral vibration of plates. This arrangement is called Active Compressional Constrained Layer Damping (ACCLD) treatment. The ACCLD represents an improvement over the conventional ACLD and MCLD, as it control the compression deflection of visco-elastic layer using the electro-magnetic actuators. Because this actuators produce significantly large amount of control force, i.e. it is possible now to control structure with large deflection or large vibration amplitude, also this design utilize viscoelastic core which can work as passive controller if the power shut down.

\section{Theory of Electromagnetic Actuator (compression type)}

The electromagnetic actuator consists of electromagnetic layer interacting with a permanent magnetic layer with air gab of height $\mathrm{s}$. The electromagnet consists of thin solenoid or coil with thickness $h$, total number of turn $N$, and number of turn per unit length $n$ as shown in Fig. 1

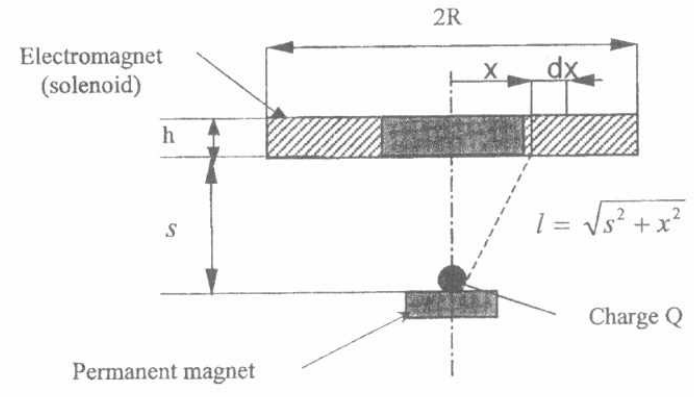

Fig. 1 Schematic drawing of electromagnetic actuator 
The interaction of forces between the solenoid and the permanent magnet can be obtained using Coulomb's law [6]. The electric field at the output of the solenoid and its effect on a charge q which, represent one pole of the permanent magnet is deduced. According to applying Coulomb's law, he force $\vec{F}$ between two charges $q_{1}$ and $q_{2}$, as shown in Fig. (2) is given by:

$\vec{F}=\frac{q_{1} q_{2}}{r^{2}} r^{\wedge} \quad$ (Gaussian system of units)

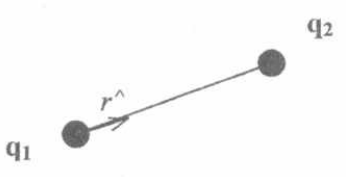

Fig 2 Two electric charges

where $q=$ charge in coulomb and $r=$ distance between charges

If there is $\mathrm{n}$ charges $q_{1}, q_{2}, \ldots \ldots \ldots q_{n}$ at a distance $\mathrm{r}_{\mathrm{n}}$ from a charge $Q$, then by using principle of superposition, the total force $\vec{F}$ on $Q$ is given by:

$$
\begin{aligned}
\vec{F} & =\vec{F}_{1}+\vec{F}_{2}+\ldots \ldots=\left(\frac{q_{1} Q}{r_{1}^{2}} r_{1}+\frac{q_{2} Q}{r_{2}^{2}} r_{2}^{2}+\ldots \ldots \ldots \ldots . . .\right) \\
& =Q\left(\frac{q_{1}}{r_{1}^{2}} r_{1}^{\wedge}+\frac{q_{2}}{r_{2}^{2}} r_{2}^{\wedge}+\ldots\right)=Q \sum_{i=1}^{n} \frac{q_{i}}{r_{i}^{2}} r_{i}^{\wedge}=Q \vec{E}
\end{aligned}
$$

where the electromagnetic field $\mathrm{E}$ is:

$\vec{E}=\sum_{i=1}^{n} \frac{q_{i}}{r_{i}^{2}} r_{i}$

The electric field depend on the shape of source charge i.e. line charge, surface charge or volume charge. The used solenoid represents surface charge, $\sigma$, with respect to a charge, $Q$, which, represent one pole of the permanent magnet, as shown in Fig 1.

The solenoid electric field can be written as:

$E(Q)=\int_{A}^{l} \frac{l^{\wedge}}{l^{2}} \sigma d A$

where $l=$ vector between the surface charge $\sigma$ and the charge $Q, l^{\wedge}=$ unit vector. Substituting into the above equation, then the vertical component of the electric field is given by:

$E(Q)=\int_{0}^{R} \frac{\sigma 2 \pi x d x}{s^{2}+x^{2}} \frac{s}{\sqrt{s^{2}+x^{2}}}$

where $2 \pi x d x=$ elementary area $\mathrm{dA}$ of solenoid with radius , R and $s=$ distance between solenoid and permanent magnets. The result of the above integration is:

$E=2 \pi \sigma\left[1-\frac{s}{\sqrt{s^{2}+R^{2}}}\right]$

in analogy with electric field the equivalent magnetic field can be represented as: 


$$
\mathrm{B}(s)=\mathrm{B}_{0}\left[1-\frac{s}{\sqrt{s^{2}+R^{2}}}\right]
$$

The force between the two magnets equal

$$
\begin{aligned}
& F_{m}=q_{m} \mathrm{~B}(s) \\
& =q_{m}\left[\mathrm{~B}\left(s+\frac{h}{2}\right)-\mathrm{B}\left(s-\frac{h}{2}\right)\right]=q_{m} h \mathrm{~B}^{\prime}(s)
\end{aligned}
$$

The term between bracket represent variation of electromagnetic field $B(s)$ with respect to $s$, i.e. it's derivative with respect to $s$

$$
\begin{aligned}
& F=-q_{m} h \mathrm{~B}_{0} \frac{R^{2}}{\left(s^{2}+R^{2}\right)^{3}} \\
& q_{m} h=M \text { represent magnetic moment of permanent magnet } \\
& B_{0} \quad \text { magnetic field inside solenoid }
\end{aligned}
$$

The magnetic field inside solenoid can be represented as [7]:

$B_{0}=\mu N I$

where $\mu$ permeability of core, $\mathrm{N}$ number of turn and I current

$$
F=-m \frac{R^{2}}{\left(s^{2}+R^{2}\right)^{\frac{3}{2}}} \quad \mu N I
$$

Actually due to vibration of the plate, the distance $s$ changes such that. $s=s \pm \Delta s$, and so the current, I changes to $I \pm \Delta I$ therefore. equation (11) is a nonlinear equation and under the following assumption the equation can be linearized: The variation of $s$ is very smal! specially after squaring its value in the above equation, one can assume that $s \cong$ cons $\tan t$, however, $m, \mu, N$, and $R$ are constants. Usuaily laser sensor is used to measure the variation in displacements by volts, which can be transferred to either current or displacement. Under the above assumption one can say that the force between two magnets will vary linearly with current. The above equation will be validated experimentally as it will be seen later.

\section{Coupling between Stiffness of Electromagnetic Actuator and Structure Stiffness}

The idea of controlling the vibration of plate can be briefly explained from the block diagram of the system transfer function, shown in Fig 3. The measured displacement, $X_{s}$, transferred into voltage, $V_{s}$, using the laser sensor gain, $\mathrm{C}_{\mathrm{s}}$, and the sensor voltage is multiplied by the amplifier gain, $G$, to get strong signal , $V_{G}$. This voltage is transferred into current, I using the coil resistance, $R$ and the obtained current will get into the electromagnetic actuator resulting in an electromagnetic force, $\mathrm{F}$ through the controller gain $\mathrm{C}_{\text {t. }}$.

The output voltage of laser sensor $V$, is related to the measured displacement by the equation $V_{s}=C_{s} X_{s}$

The amplified laser sensor voltage signal is 
$V_{G}=G V_{s}$

The current voltage relationship is

$I=\frac{V_{G}}{R}$

Substituting value of $V_{G}$ from equation (13) into (14)

$I=\frac{G V_{s}}{R}=\left(\frac{G C_{s}}{R}\right) X_{s}$

From equation (11) the force current relationship can be assumed as $F_{c}=C_{f} I$

Substituting value of current from equation (15) into equation (16) leads to force displacement relationship

$F_{c}=C_{f}\left(\frac{G C_{s}}{R}\right) \quad X_{s}=C X_{s}$

where. $\mathrm{F}$ and $\mathrm{C}_{\mathrm{f}}$ are actuator force and actuator gain respectively.

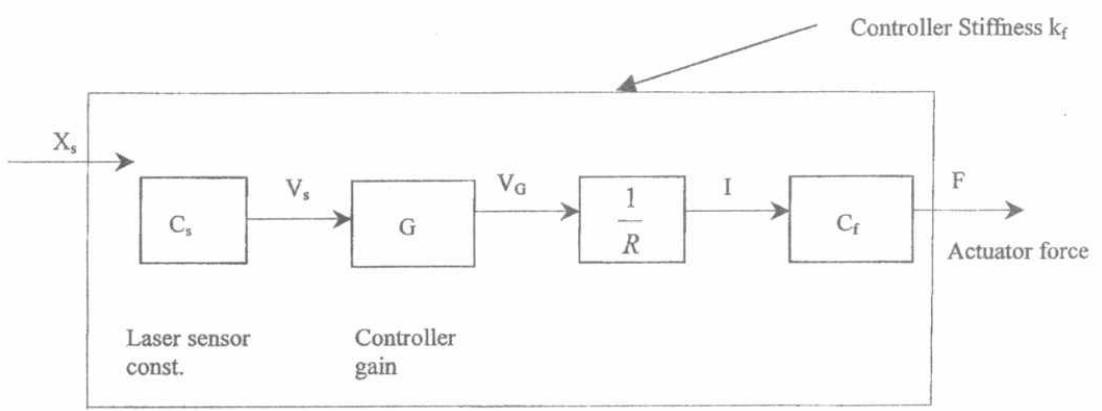

Fig. 3 Stiffness due to electromagnetic controller $K_{f}$

\section{Controller Stiffness Matrix Contribution to the Structural Stiffness Matrix K}

Through this analysis a clamped-clamped plate with ACCLD patch is used. The plate/ACCLD system is divided into $\mathrm{n}$ quadrilateral elements. Each element has 4 nodes and each node has six degrees of freedom as $w_{1}, w_{1, x}, w_{1, y}, w_{3}, w_{3, x}, w_{3, y}$, where $w_{1}, w_{1, x}, w_{1, y}$ are deflection, slope (with respect to $\mathrm{x}$ and $\mathrm{y}$ ) of $1^{\text {st }}$ layer and $w_{3}, w_{3, x}, w_{3, y}$ are deflection, slope (with respect to $x$ and $y$ ) of $3^{\text {rd }}$ layer. The equation of motion of the whole structure is given by: $[M] \ddot{X} \mid+\lceil K\rceil X\rceil=-\left\lceil F_{c}\right\rceil$

From equation (17), $F_{c}=C_{f}\left(\frac{G C_{s}}{R}\right) X_{s}$ the whole values between the previous brackets are constants and considered as $\mathrm{C}$. Hence, $\mathrm{F}_{\mathrm{c}}$ can be written as: 


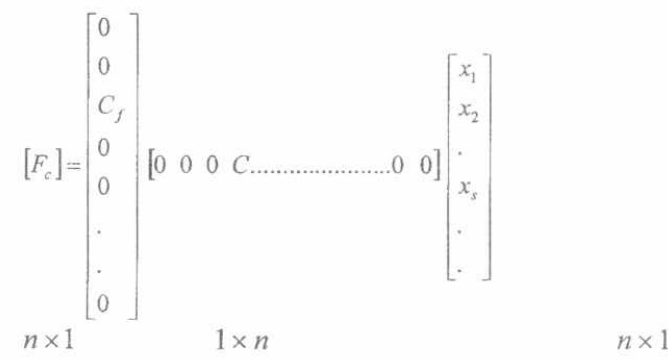

, $\mathrm{F}_{\mathrm{c}}$, can be represented in matrix form as

$\left[F_{c}\right]=\left[k_{f}\right][x]$

The constant of equation (17) now transferred into $n \times n$ matrix, representing the stiffness matrix due to controller force, $\mathrm{K}_{\mathrm{f}}$.

$[M] \ddot{x} \mid+\left[K+K_{f}\right]\lceil X]=[0]$

The above mentioned theory augmented into the compression finite element model to transfer the passive compression model (CCLD) into an active compression model (ACCLD).

\section{Dynamic Performance of the Electromagnetic Actuator}

The theoretical prediction of the performance of the actuator is validated experimentally in this section. The actuator consists of a viscoelastic high-density foam sandwiched between an electromagnet and a permanent magnet (Neodymium Blocks) (type NB2518181475-35) as shown in Fig. 4. The electromagnetic force of the coil acts along the same line of action as that of the magnetic force of permanent magnet. The operating principle of the actuator depends on the attraction or repulsion forces that exist between the two magnets. For controlling the first mode of vibration, the actuator is placed at the middle of fixed-fixed plate. Hence, as the plate moves up the polarity of the electromagnet is designed to be the same as permanent magnet, $\mathrm{N}-\mathrm{N}$, which leads to repulsion force pushing the plate down. But, once the plate starts going down, the polarity changes to be S-N and attraction force is generated pulling the plate up. Actually the laser sensor used here is utilized to detect the vibration and provide the negative feedback signal necessary to control the electromagnetic actuator. The viscoelastic rubber-foam is used as passive means for vibration damping.

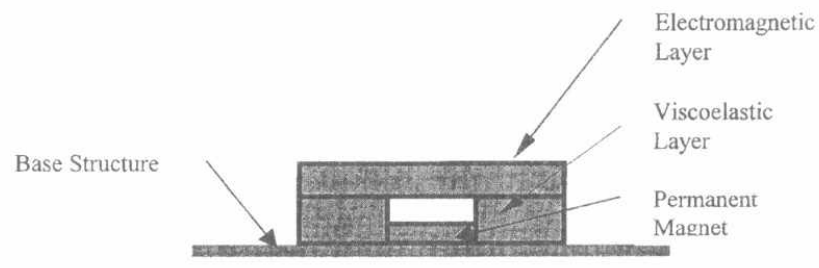

Fig. 4 Schematic drawing of ACCLD patch 


\subsection{Static Deflection - Current Characteristic}

Experiments are carried out to determine the static deflection, current characteristics of the electromagnet. The test specimen consists of a viscoelastic material sandwiched between a permanent magnet and an electromagnet. The polarity of electromagnet is arranged such that it makes attraction force with the permanent magnet. The experimental set-up consists of a test specimen connected to an AC current supply. The current supplied to the actuator is connected as an input to an analog to digital converter (AVD). The laser sensor output is connected to the A/D converter. The outputs from the A/D converters are connected to the computer. A schematic drawing of the experimental set-up is shown in Fig. 5. The static displacement-current characteristics of ACCLD are displayed in Fig. 6. From this figure, it is shown that the current increases with the electromagnetic force. The characteristics are almost linear as is predicted by the theoretical model.

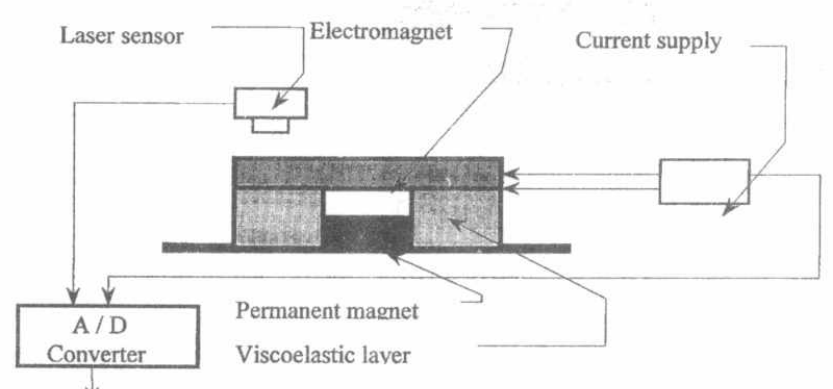

Computer

Fig. 5 Schematic drawing for experiment used to measure deflection-current characteristics

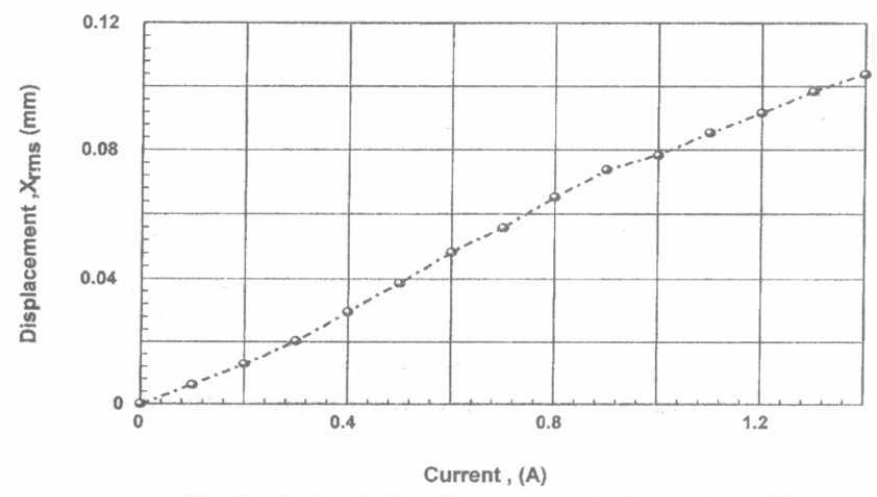

Fig 6 Static deflection-current characteristics 


\subsection{Deflection-Frequency Characteristics for Constant Current}

Sweep experiments have been conducted to sweep the current as a function of frequency in order to obtain the Defiection-Frequency characteristics for different current ievels. The AC current supply is connected as input to the channel A of the FFT analyzer (model ONO SOKKI, CF910), while the output from laser serisor was connected to channel B of the analyzer as shown in Fig. 7. In this manner the transfer furiction of the actuator can be obtained. The vertical deflection-frequency characteristics are shown in Fig. 8 for low and high gains. From this figure, it is noticed that the characteristics is nearly constant up to frequency of $70 \mathrm{~Hz}$. Actuator resonance at about $125 \mathrm{~Hz}$ is evident. Such characteristic is particularly suitable for controlling the first three modes of vibration of the plate, which are $<50 \mathrm{~Hz}$.

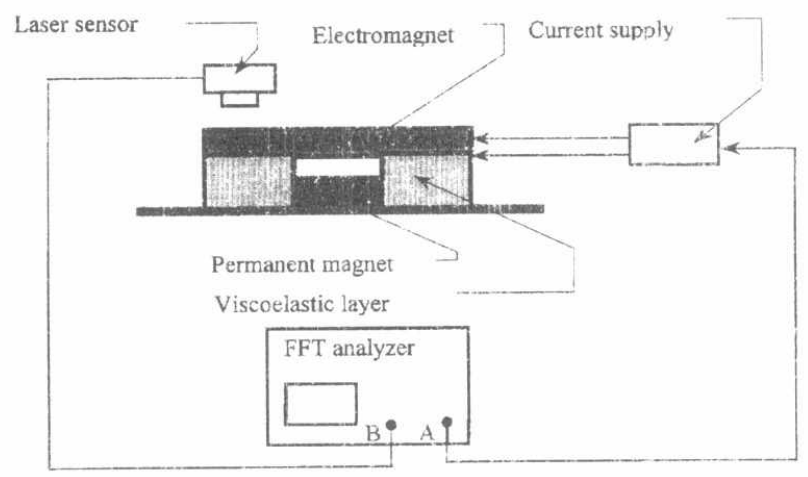

Fig. 7 Schematic drawing for experiment used to measure deflection-frequency characteristics

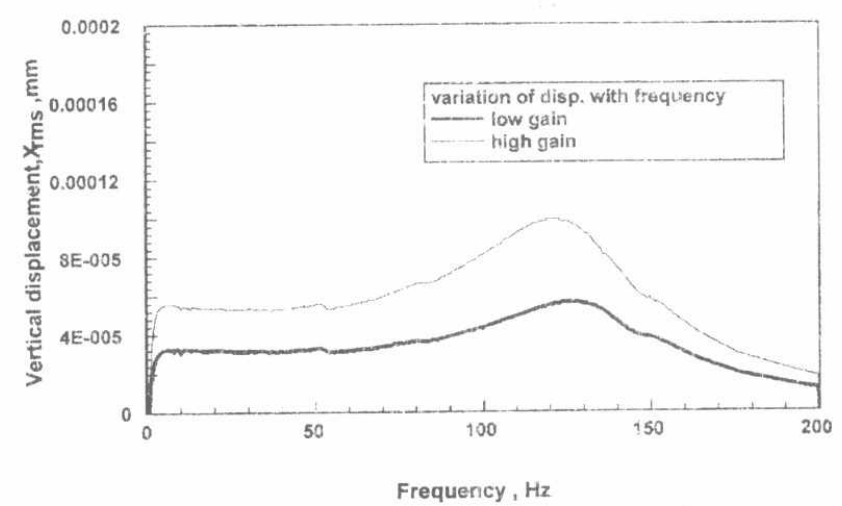

Fig. 8 Deflection-frequency characteristics 


\subsection{Measuring of Electromagnetic Force}

Electromagnetic force of the actuator is monitored using a load cell (model ALD-MINIUTC-50, A.L. Design, INC., Buffalo, NY), as shown in Fig 9. The load cell has sensitivity of $2.1 / 22.24 \mathrm{mV} / \mathrm{N}$. When the current is passed through the electromagnet, an attraction force is generated. As the input current is increased the attraction force is found to increase, almost in a linear fashion as shown in Fig 10.

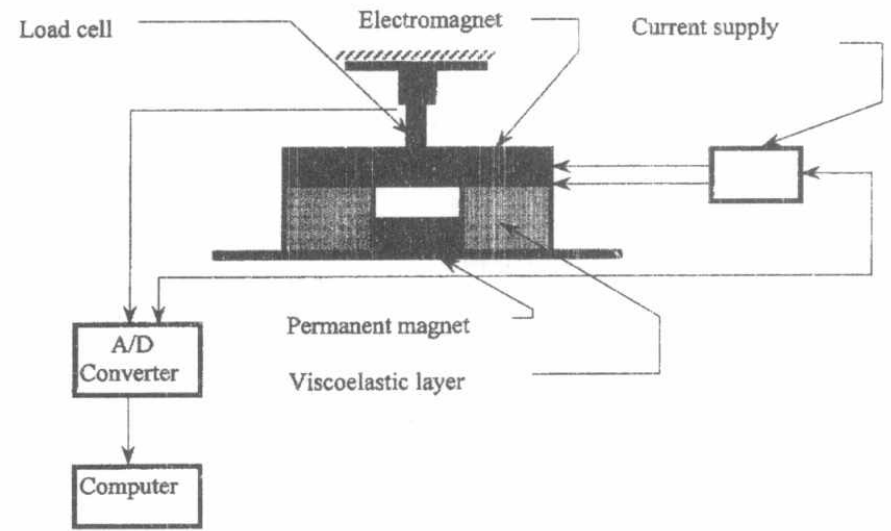

Fig. 9 Experiment used to determined the variation of electromagnetic force with current

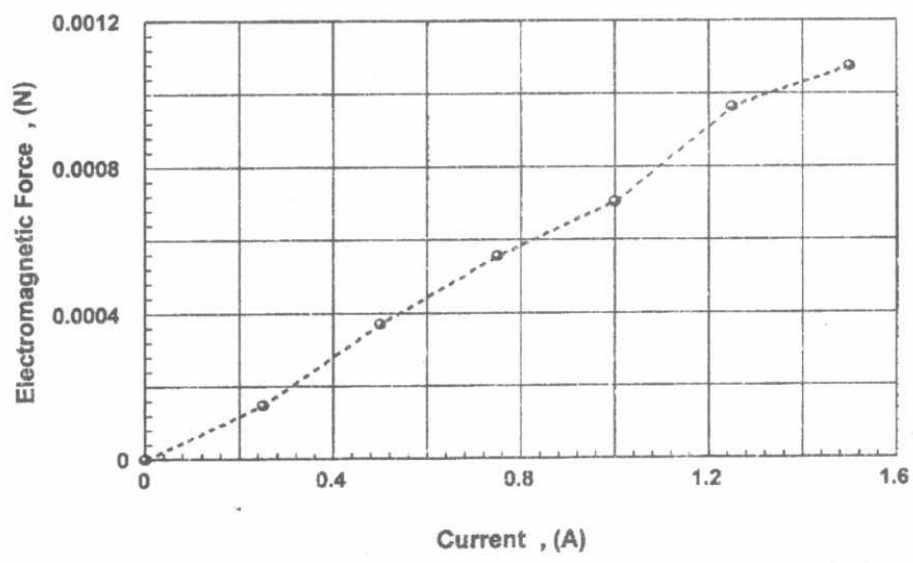

Fig. 10 Electromagnetic magnetic force-current characteristics 


\subsection{Electromagnetic Force - Frequency characteristics for Constant Currents}

A dynamic FFT analyzer is used to generate the frequency sweep. The current supplied to the actuator is connected to the channel B of the FFT analyzer as input, while the output from load cell is connected to the channel A, as shown on Fig. 11. According to this arrangement one can measure the electromagnetic force-frequency characteristics of the actuator at constant current as shown in Fig. 12. The transfer function between the current supply signal and the load cell signal is automatically determined as magnitude ratio (dB) and phase angle (deg.). Fig. 13 shows plot of the transfer function as magnitude and phase for electromagnetic actuator with frequency range from $0-200 \mathrm{~Hz}$. Moreover Fig. 14 shows the magnitude and phase of the actuator transfer function for frequency range from $0-500 \mathrm{~Hz}$

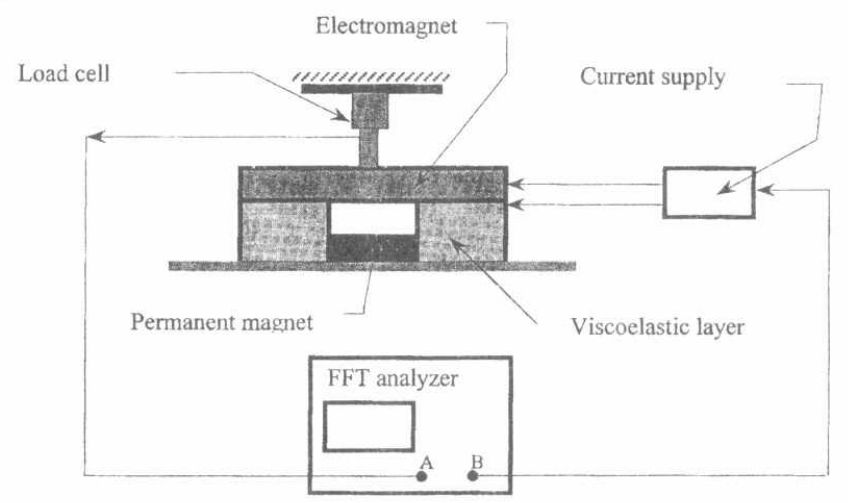

Fig. 11 Experiment setup used for measuring variation of electromagnetic force with frequency

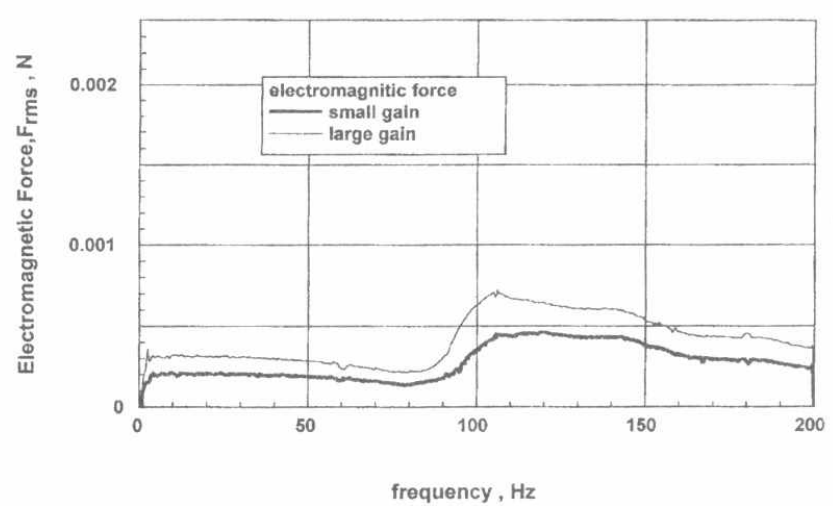

Fig. 12 variation of electromagnetic force with constant current as a function of frequency 


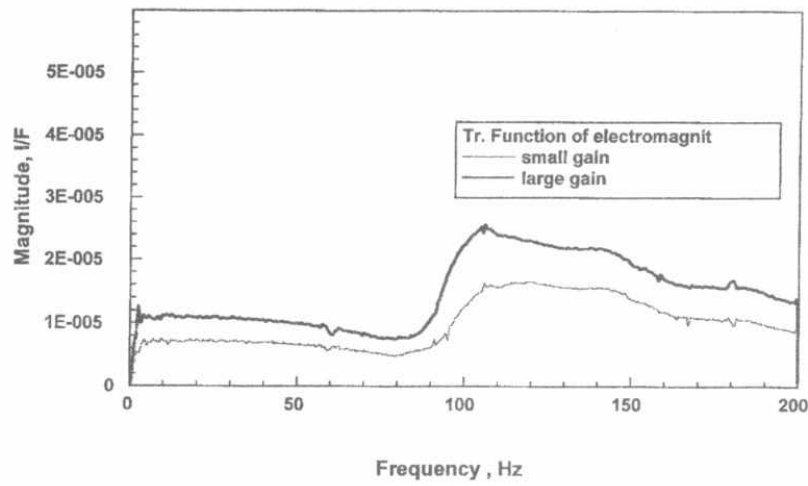

a)

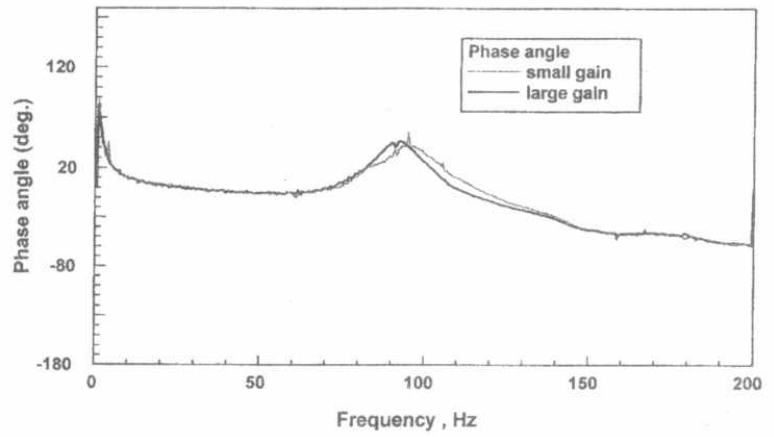

b)

Fig. 13 Transfer function of electromagnet

$\begin{array}{ll}\text { (a) magnitude } & \text { (b) phase }\end{array}$ 


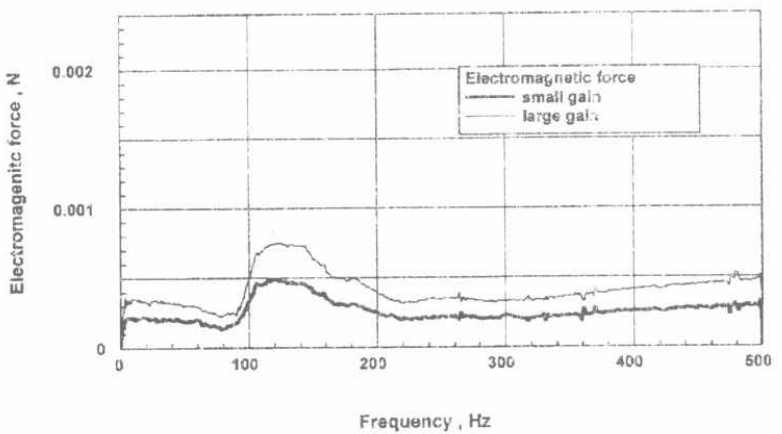

a)

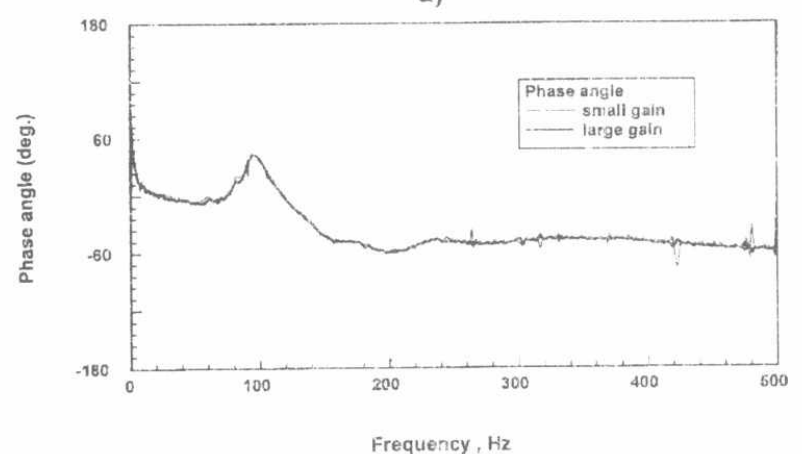

b)

Fig. 14 Transfer function of electromagnet - frequency range $0-500 \mathrm{~Hz}$ $\begin{array}{ll}\text { (a) magnitude (b) phase } & \end{array}$

\subsection{Electromagnetic Controlier}

The electromagnetic actuator is bonded to the center of fixed-fixed plate to control the first mode. The treatment consists of the ACCLD with a viscoelastic material sandwiched between a permanent magnet and an electromagnet. The poles of the electromagnet change from north to south when the plate vibrate to generate either attraction or repulsion forces when the electromagnet interacts with the permanent magnet.

\subsection{Active Compressional Constrained Layer Damping (ACCL.D)}

The active compression constrained layer damping (ACCLD) is a new class of treatment is developed to achieve high authority control force. Due to the attraction or repulsion forces generated between the two magnets, a compression or expansion of the viscoelastic layer will occur. This leads to vibration damping due to energy dissipation in the viscoelastic material. 


\subsection{Experimental Set up}

The tested square plate is mounted with clamped edges . A shock test is carried out on the clamped plate to determine its natural frequencies. Table 1 shows the modes of vibration of the plate within a range from $0-200 \mathrm{~Hz}$. A single ACCLD patch is bounded to its geometric center. The frequency generates in the dynamic FFT analyzer is used to excite the plate using the sweep function. A sine sweep experiment is carried out to determine the effect of ACCLD on vibration damping of plate The output from the sweep function is magnified using a power amplifier (model 6260, JBL, UREI Electronic Products) which is used to derive the speaker. The plate vibration is monitored using laser sensor type (LA4Ohr) Also, the sound radiation from the plate is monitored using a $1 / 2$ inch microphone $(B \& K$ instruments, type 4165) The output of either the laser sensor or the microphone is used to generate the necessary control action. This action is developed using a filter (WAVEETEK dual Hi / Lo model 432), which is connected to a low noise amplifier (type, AM5), and another power amplifier (Wilcoxon Research type, PA7C), in series. The ACCLD arrangement is shown in Fig. 15 for laser feedback.. The laser sensor is used to feedback the control voltage. Fig. 16 shows the plate vibration with feedback from the laser sensor itself for three different gains. Fig. 17 shows the sound pressure level for the same three gains, with feedback from the laser sensor. Tables 2 and 3 give the frequency and damping ratio of the first mode of vibration for the plate with a single patch of ACCLD. Table 2 gives the modal parameters using the laser sensor, while Table 3 gives these parameters using the microphone signal. In these tables as the controller gain increases, the damping ratio increases and the natural frequency decreases.

Table 1 Modes of vibration of plate using impact hammer

\begin{tabular}{|l|l|l|l|l|l|}
\hline & $1^{\text {st }}$ & $2^{\text {nd }}$ & $3^{\text {rd }}$ & $4^{\text {th }}$ & $5^{\text {th }}$ \\
\hline Freq. Range 0-100 Hz & 19.0 & 59.0 & 59.0 & 78.75 & N/A \\
\hline Freq. Range 0 -200 Hz & 18.5 & 57.75 & 57.75 & 78.0 & 133.5 \\
\hline
\end{tabular}

Table 2 Effect of gain on fundamental frequency and damping ratio using laser sensor

\begin{tabular}{|l|l|l|}
\hline Controller gain, $k_{d}$ & $\begin{array}{l}\text { First mode frequency , } \\
H z\end{array}$ & Damping ratio \\
\hline 0 & 19.18 & 0.0128 \\
\hline 89.44 & 19.19 & 0.0179 \\
\hline 184.75 & 18.8 & 0.0311 \\
\hline 362.17 & 18.52 & 0.0769 \\
\hline
\end{tabular}

Table 3 Effect of gain on fundamental natural frequency and damping ratio using microphone output

\begin{tabular}{|l|l|l|}
\hline Controller gain, $\mathrm{k}_{\mathrm{d}}$ & First mode frequency, $\mathrm{Hz}$ & Damping ratio \\
\hline 0 & 19.19 & 0.0125 \\
\hline 89.44 & 19.01 & 0.0181 \\
\hline 184.75 & 18.83 & 0.0316 \\
\hline 362.17 & 18.57 & 0.0805 \\
\hline
\end{tabular}




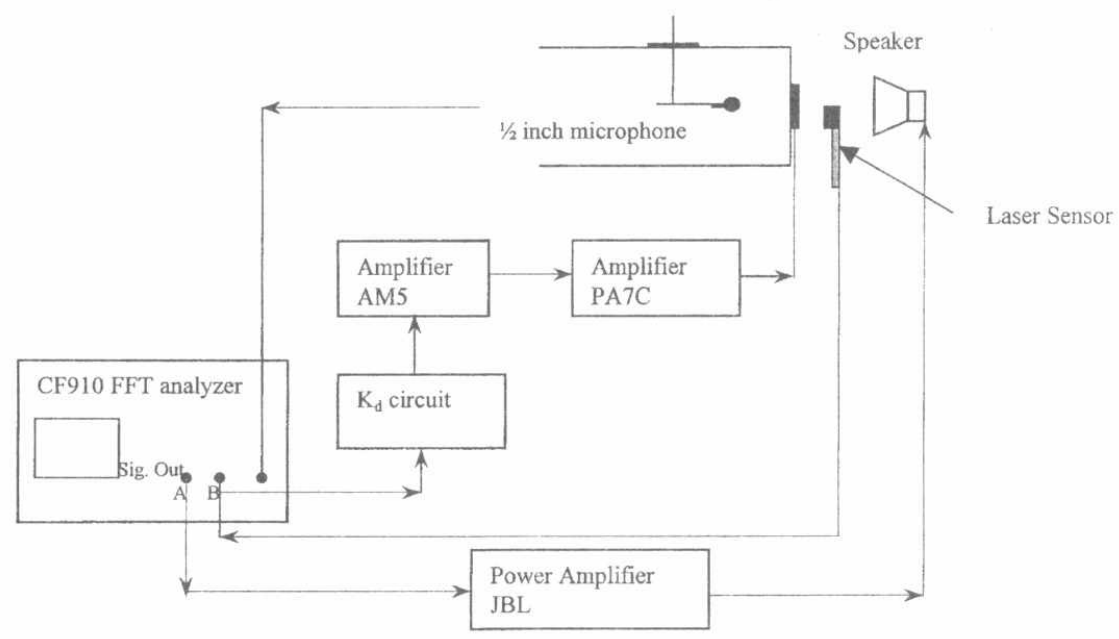

Fig. 15 schematic drawing of the magnetic constrained layer damping experiment.

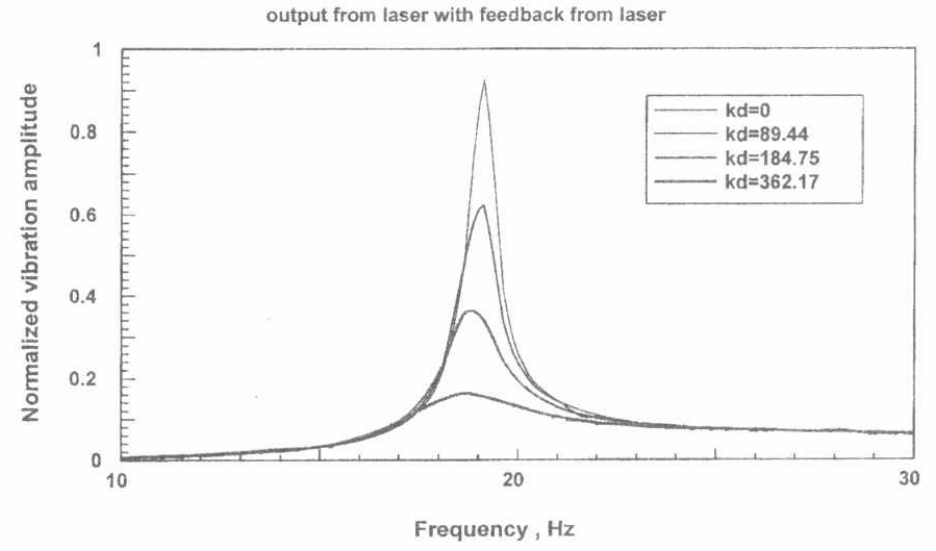

Fig. 16 Normalize vibration amplitude with laser feedback and open cavity . 


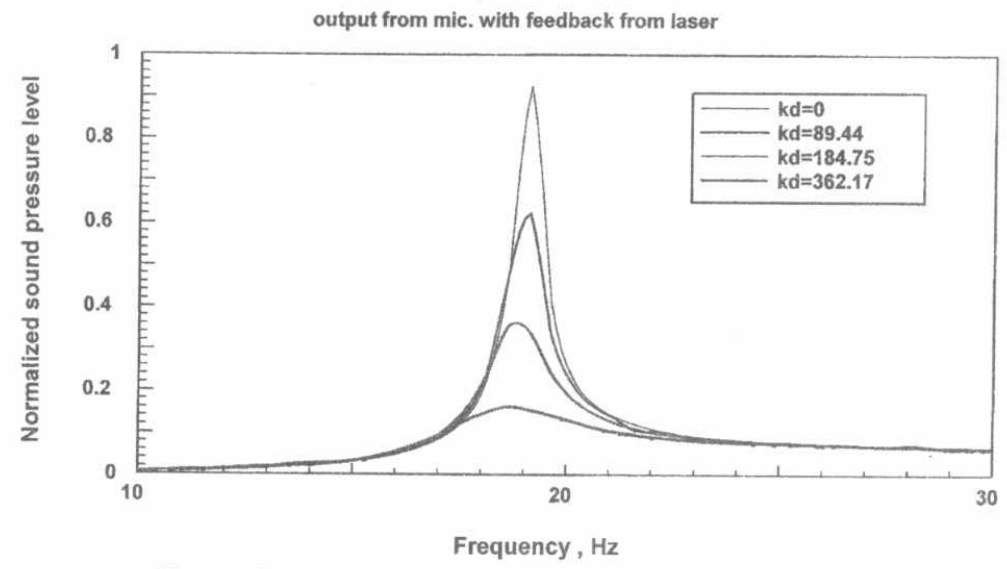

Fig 17 Sound pressure level of open cavity with laser feedback at different gain.

\section{Conclusions}

This paper has presented a theoretical and experimental procedure for the magnetic electromagnetic controller. The dynamic performance and characteristic of the controller was obtained. The variation of displacement and force with current are almost linear as expected theoretically. The controller characteristics was nearly constant up to frequency of $70 \mathrm{~Hz}$. Actuator resonance was about $125 \mathrm{~Hz}$ then it became nearly constant again up to $500 \mathrm{~Hz}$. Such characteristic was particularly suitable for controlling the first three modes of vibration of the plate, which are $<50 \mathrm{~Hz}$. The potential of using the electromagnetic actuator for damping the lateral vibration of clamped-clamped plate was shown. Using the viscoelastic material during the design of the controller works as a passive means for vibration damping. Good agreement was obtained between the theoretical and experimental results for force-current relationship.

\section{References}

1 Omer, A. and Baz, A. "Vibration Control of Plates Using Electromagnetic Compressional Damping Treatment", Proc. Of the $17^{\text {th }}$ Biennial Conf. On Vibration and Noise", Las Vegas, Nevada, Sept. 1999.

2 Baz ,A. and J. Ro, " Performance Characteristics of Active Constrained Layer Damping", Shock and Vibration Journal, Vol.2, No.1, pp.33-42, 1995a

3 Zheng, K. W., "Moment-Type Electromagnetic Actuator and Its Application to Plate Deflection Controls", Int. J. of Applied Electromagnetic and Mechanics, Vol. 6, pp. 15-35, 1995.

4 Frangos, C. and Yavin Y., " Current Controller Design for an Electromagnetic Actuator Using an On-line Parameter Optimization Approach", IEEE Transactions on Industrial Electronics, Vol. 38. No.1, pp.48-50, February 1991.

5 Baz, A. "Magnetic Constrained Layer Damping", Eleventh Symposium on Structural Dynamic \& Control, Blacksburg, VA-May 12-14. 1997. 
6 Griffith, D. J, "Introduction to Electromagnetic", Prentice Hall, 1995

7 Vanderlinde ,J., " Classical Electromagnetic Theory", J. Wiley and Sons, 1993

8 Moon, F., "Magneto-Solid Mechanics", J. Wiley \& Sons, New York, 1984.

9 Yuan,K. Y. , "Finite Element Analysis of Magneto-Elastic-Plate Problems ", Ph.D. Dissertation, Cornell university, August 1981.

10 Nashif, A., D. Jones and J. Henderson, "Vibration Damping", J. Wiley \& Sons, New York, 1985

11 Ferry, J. D., "Viscoelastic Properties of Polymers ", Wiley, New York, 1961.

12 Sun, C. T and Lu, Y. P. "Vibration Damping of Structural Elements" PrenticeHall 1995

13 Bathe, K. J., "Finite Element Procedures in Engineering Analysis", Prentice-Hall, 1982.

14 Cook ,R. D.,. " Concepts and Applications of Finite Element Analysis", John Wiley \& Sons, 1989. 